\title{
Las cardiopatías congénitas en el 2018
}

\author{
Clara Vázquez-Antona, ${ }^{1}$ Carlos Alva-Espinosa, ${ }^{2}$ Lucelli Yáñez-Gutierrez ${ }^{3}$ y Horacio Márquez-González ${ }^{3}$
}

${ }^{1}$ Secretaría de Salud, Instituto Nacional de Cardiología, Departamento de Ecocardiografía; ${ }^{2}$ Hospital Ángeles del Pedregral; ${ }^{3}$ Instituto Mexicano del Seguro Social, Centro Médico Nacional Siglo XXI, Hospital de Cardiología. Ciudad de México, México

\section{Resumen}

Se presenta una actualización de los avances más relevantes de la ecocardiografía tridimensional para el diagnóstico de las cardiopatías congénitas. Se analiza la evolución de su tratamiento y se describen los resultados obtenidos en los últimos años. En el tema de la mujer embarazada con cardiopatía congénita se subrayan los cambios funcionales y estructurales durante el embarazo, así como los riesgos por considerar y la importancia del trabajo en equipo para tratar a estas enfermas. Finalmente se aportan datos de la situación en México y las medidas recomendadas para mejorarla.

PALABRAS CLAVE: Cardiopatías congénitas. Diagnóstico ecocardiográfico. Embarazo.

\begin{abstract}
We present an update of the most relevant advances of three-dimensional echocardiography for the diagnosis of congenital heart diseases. The evolution of its treatment is analyzed, showing the results obtained in recent years. The functional and structural changes experienced in women with congenital heart disease during pregnancy are discussed, as well as the main health risks and the importance of teamwork in healthcare to treat these patients. Finally, we provide a review on the current situation in Mexico and outline some improvement recommendations.
\end{abstract}

KEY WORDS: Congenital heart diasease. Echocardiographic diagnosis. Pregnancy.

Gac Med Mex. 2018;154:698-711 Disponible en PubMed www.gacetamedicademexico.com 


\title{
Ecocardiografía tridimensional en cardiopatías congénitas
}

\author{
Clara Vázquez-Antona
}

La compleja anatomía y los sofisticados mecanismos de función de las estructuras cardiacas en los pacientes con cardiopatía congénita (CC) requieren que la aplicación de las técnicas ecocardiográficas como método diagnóstico cubran dos aspectos básicos: morfología y función. La ecocardiografía bidimensional (Eco2D) y Doppler es hasta este momento una herramienta diagnóstica útil y en muchos casos suficiente para la evaluación morfológica y hemodinámica. Una de las limitaciones de la Eco2D radica en que se realiza en un solo plano, por lo que se requiere la obtención de imágenes en diferentes aproximaciones, lo que obliga a realizar una reconstrucción mental para su mejor entendimiento.

La ecocardiografía tridimensional (Eco3D) valora el corazón en sus tres dimensiones, ya sea en una vista de conjunto o bien con planos convencionales y no convencionales. El beneficio de la reconstrucción tridimensional (3D) del corazón con defectos congénitos radica en el potencial de mostrar objetivamente la anatomía y las complejas relaciones entre las diferentes estructuras cardiacas, logrando imágenes orientadas en forma ilimitada como las vistas "de superficie" de las válvulas atrioventriculares (AV) y ventriculoarteriales, así como del septum interatrial e interventricular, además de la valoración de la función ventricular izquierda y derecha y de las cardiopatías de fisiología univentricular. ${ }^{1} \mathrm{~A}$ diferencia de la reconstrucción 3D en cardiopatía adquirida, los pacientes con CC complejas pueden tener alteraciones en la posición del corazón, situs, conexiones y relaciones de las estructuras, lo que implica un mayor reto que en los pacientes con patología adquirida.

El uso de la Eco3D para el estudio rutinario de las CC requiere una curva de aprendizaje y exige entrenamiento específico, ya que además del conocimiento sobre CC y su tratamiento, la capacitación debe incluir la adquisición y posprocesamiento de los datos 3D para demostrar la competencia en la evaluación morfológica y cuantificación de la función cardiaca. ${ }^{2,3}$ Se debe aprender a reconocer y evaluar las vistas del corazón que son factibles con 3D, incluidas las vistas de las válvulas y septos.

\section{Antecedentes}

Dekker et al. iniciaron en 1974 la imagen cardiaca tridimensional utilizando un brazo mecánico articulado "manos libres" para obtener las imágenes transtorácicas para su posterior procesamiento. En 1993, Pandian y Roelandt introdujeron la Eco3D dinámica con transductor transesofágico multiplanar, en la que la reconstrucción iniciaba a partir de la adquisición de imágenes planares bidimensionales obtenidas en sincronía con el ciclo cardiaco y los movimientos respiratorios, las cuales eran almacenadas en una base de datos independiente. A inicios de la década de 1990, von Ramm et al. ${ }^{4}$ desarrollaron el primer equipo ecocardiográfico tridimensional en tiempo real, el cual ha tenido mejoras en el diseño e ingeniería hasta los equipos que utilizamos actualmente tanto para abordajes transtóracicos como transesofágicos.

En México, la experiencia con Eco3D inició en el Departamento de Ecocardiografía del Instituto Nacional de Cardiología "Dr. Ignacio Chávez" en 1999 con la Eco3D off line por Vargas-Barrón et al. ${ }^{5}$ En 2002 de reportaron los resultados de los primeros 54 pacientes con $\mathrm{CC}$ a quienes se realizó reconstrucción 3D a partir de imágenes bidimensionales secuenciales transesofágicas con posprocesamiento. En esta experiencia inicial se compararon las imágenes bi y tridimensionales y se correlacionaron con especímenes anatómicos equivalentes para valorar su correspondencia.

Para entonces se demostró que la reconstrucción 3D de las cardiopatías era posible en todos los casos, confirmando que definía detalles anatómicos adicionales a los obtenidos por $2 \mathrm{D}$, ya que se lograban reconstrucciones desde vistas no convencionales, como las observadas desde los atrios o ápex de las conexiones atrioventriculares, y vistas de superficie tanto del septum interatrial como interventricular; aun cuando las adquisiciones consumían tiempo, se obtenían imágenes que proporcionaban valiosa información adicional. ${ }^{6}$

En 2005 se inició la experiencia con ecocardiografía transtorácica 3D en tiempo real, método que fue fácilmente aplicado a los pacientes pediátricos, ya que las ventanas acústicas son favorables y la información morfológica es indispensable para el entendimiento de las CC; en 2011 se comenzó con la Eco3D transesofágica en tiempo real. Actualmente la mayoría de los laboratorios de ecocardiografía de centros que manejan 
pacientes con CC han integrado esta tecnología como método diagnóstico complementario.

\section{Aplicaciones clínicas de la ecocardiografía tridimensional}

La aplicación clínica de la reconstrucción 3D del corazón con defectos congénitos abarca principalmente tres áreas: visualización de la morfología, cuantificación volumétrica de las cámaras y flujos cardiacos y la guía en procedimientos invasivos transcateterismo y de valoración intraoperatoria. La reconstrucción 3D en tiempo real es posible desde las aproximaciones transtorácica y transesofágica (ETE). La limitación de la ETE 3D es que la sonda solo puede ser utilizada en pacientes con más de $20 \mathrm{~kg}$ de peso. La adquisición de datos puede ser en dos formas:

1. En tiempo real "live 3D" mediante la imagen multiplanar simultánea, que muestra imágenes bidimensionales en dos planos ortogonales simultáneos, el modo 3D en tiempo real y la amplificación 3D o zoom 3D.

2. Multilatido, coordinada con el electrocardiograma, en la que se pueden obtener volúmenes completos o de ángulo amplio y volumen completo con Doppler color para posprocesamiento.

Las aportaciones de la Eco3D pueden estratificarse en tres grupos:

1. Información equivalente a la de los estudios con Eco2D.

2. Hallazgos nuevos no vistos en Eco2D, pero que no modifican en forma crítica la decisión terapéutica.

3. Información anatómica adicional útil en la toma de decisiones terapéuticas.

Los dos primeros grupos permiten un mejor entendimiento de la anatomía con imágenes "agradables" y perspectivas anatómicas interesantes y son más útiles desde el punto de vista académico que terapéutico y para mejorar la comunicación con clínicos y cirujanos.

El tercer grupo brinda mayor beneficio clínico, determinado por la capacidad de definir los defectos desde las vistas de superficie atrial, ventricular y valvular con información adicional útil en las decisiones terapéuticas. En este grupo se encuentran las imágenes obtenidas en las cardiopatías con formas complejas de defectos septales atriales y ventriculares, canal atrioventricular común (Figura 1), anomalías congénitas de la válvula mitral (VM) y alteraciones de la conexión ventrículo arterial, como doble cámara de salida de ventrículo derecho, así como función ventricular
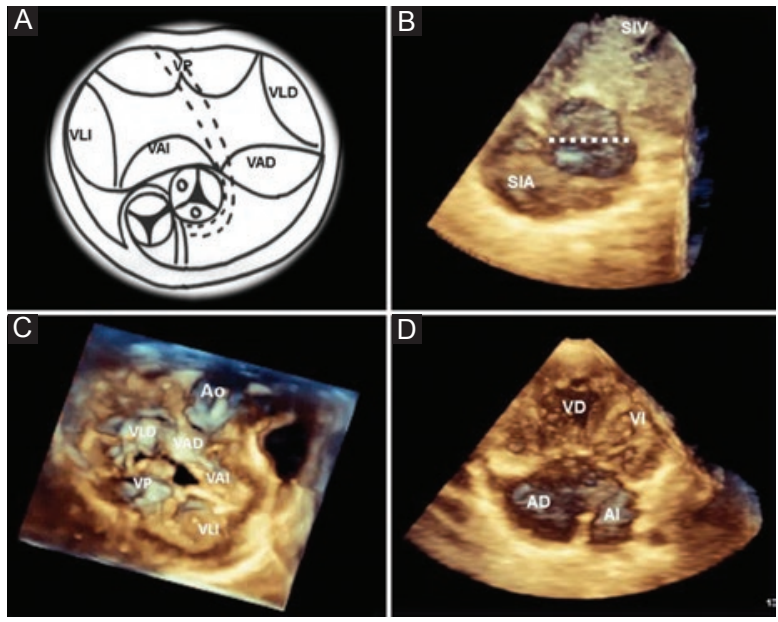

Figura 1. Imágenes transtorácicas de reconstrucción tridimensional de un paciente con canal atrioventricular común y esquema $(A)$. Se muestran las vistas no convencionales desde la vista septal izquierda $(B)$, vista de superficie atrial (C) y vista de cuatro cámaras, donde se observa la válvula atrioventricular común y los defectos septales atrial y ventricular (D). En B observamos los defectos ventricular $y$ atrial de tipo ostium primum "de frente"; la línea punteada marca en nivel del septum AV, en este caso ausente. En $C$ se observan las cinco valvas con sus componentes valvulares derecho e izquierdo y las válvulas puente anterior y posterior y su correspondencia con el esquema en $A$. VAI = valva anterior izquierda, $V L I=$ valva lateral izquierda, $V P=$ valva posterior, $V L D=$ valva lateral derecha, $V A D=$ valva anterior derecha, $A o=$ aorta, $V D=$ ventrículo derecho, $V I=$ ventrículo izquierdo, $A D=$ aurícula derecha, $S I V=$ septum interventricular, $S I A=$ septum interauricular.

izquierda en cardiopatías donde el ventrículo izquierdo ha perdido su geometría. Aproximadamente $35 \%$ de los estudios corresponde a este último grupo. ${ }^{8}$

Recientemente se publicó un documento de consenso $0^{9}$ que muestra una revisión de la aplicación óptima de Eco3D en CC, incluidas las consideraciones técnicas, la aplicación a diferentes lesiones, la guía de procedimiento y la evaluación funcional; establece que se requiere una orientación consistente e intuitiva de las imágenes 3D, conforme un mejor enfoque "anatómico", en el que se visualice el corazón en la misma orientación que una persona en posición vertical. Con este enfoque, las estructuras posicionadas superiormente se mostrarán en la parte superior de la imagen. Las "vistas quirúrgicas" se han utilizado para describir proyecciones 3D más parecidas a las vistas durante una cirugía, en donde las imágenes se proyectan como si el paciente estuviera acostado en posición supina con el cirujano principal operando desde el lado derecho del paciente.

Se debe tener en cuenta que la realización de la Eco3D debe adaptarse a la cardiopatía. A continuación se mencionan algunas aplicaciones en la que se ha demostrado mayor utilidad. 

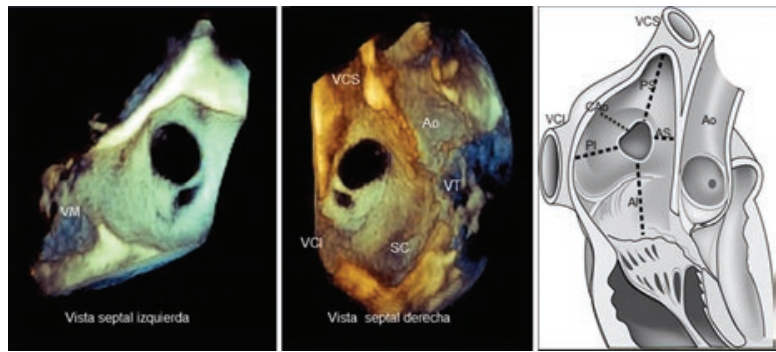

Figura 2. Imágenes ecocardiográficas tranesofágicas $3 D$ desde las vistas de superficie septal atrial izquierdo $(A)$ y derecho $(B)$, donde se observa un defecto interatrial y su relación con estructuras adyacentes. En el esquema (C), las líneas punteadas marcan los bordes: los posteriores están en relación con las venas cavas y los anteriores con la aorta y las válvulas atrioventriculares. $V M=$ válvula mitral, $V C S=$ vena cava superior, $\mathrm{VCl}=$ vena cava inferior, $A o=$ aorta, $V T=$ válvula tricúspide, $S C=$ seno coronario.

\section{Comunicación interauricular}

En los defectos del septum interatrial se logra la visualización completa del defecto para definir el tipo, localización, número y morfología, medición de los bordes del defecto y la distancia a otras estructuras (Figura 2). Estos detalles anatómicos son importantes cuando la opción de tratamiento es intervencionista, con la cual se puede determinar qué defectos son susceptibles de cierre o los que pudieran representar dificultad técnica por las diversas variantes anatómicas. ${ }^{10}$

En la actualidad se están desarrollando diferentes técnicas que permiten la creación de imágenes híbridas o la fusión de imágenes para obtener al mismo tiempo más información anatómica y funcional. ${ }^{11} \mathrm{En}$ el mercado existen equipos que fusionan imágenes de rayos $X$ y ecocardiografía $2 D$ y $3 D$ en tiempo real. Este sistema puede utilizarse como guía de procedimientos en cardiopatía estructural, como el cierre percutáneo de comunicaciones interauriculares o foramen oval permeable. Durante el procedimiento permite marcar estructuras mediante puntos o elipses en una de las imágenes ecocardiográficas; estos marcadores aparecen de forma automática y simultánea en las demás imágenes, con lo que se tiene la posibilidad de ver una estructura marcada en todas las imágenes ecocardiográficas y la imagen de fluoroscopia al mismo tiempo.

\section{Patología mitral congénita}

A diferencia de las alteraciones mitrales del adulto, la enfermedad congénita de la VM por lo general es compleja y afecta a más de un componente valvular dependiendo de la alteración embriológica. En estos casos, las vistas "de superficie", tanto desde la aurícula

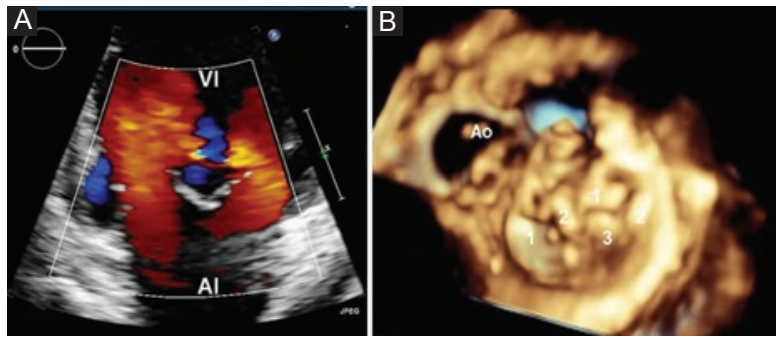

Figura 3. Imágenes ecocardiográficas transtorácicas bi y tridimensionales donde se observa un doble orificio mitral por bandeleta central (A). Obsérvese el detalle anatómico que se logra con la reconstrucción tridimensional desde la vista de superficie atrial $(B)$, donde se puede confirmar que el orificio medial tiene dos valvas, mientras que en el lateral se observan tres valvas, señaladas con números. $A l=$ aurícula izquierda, $\mathrm{VI}=$ ventrículo izquierdo, $\mathrm{Ao}=$ aorta.

izquierda como desde el ventrículo izquierdo, ofrecen una evaluación detallada de la movilidad de las valvas y de la fusión de las comisuras. ${ }^{12}$ La vista desde el ventrículo izquierdo evalúa el aparato subvalvular, definiendo con claridad la presencia de un único músculo papilar y la inserción de todas las cuerdas tendinosas en ese músculo, como la VM en paracaídas. En la VM en hamaca se demuestra que la obstrucción es causada por una alteración en el aparato subvalvular mitral y la inserción directa de las valvas sobre los músculos papilares. Desde la vista de superficie del atrio izquierdo se puede definir con precisión la membrana supravalvular mitral, el doble orificio mitral (Figura 3) y la hendidura de la valva anterior de la mitral.

EI ETE 3D ofrece mejor calidad de imagen por su mayor resolución temporal y espacial en relación con las obtenidas en 3D por vistas transtorácicas, está indicado cuando se requiere mejor definición anatómica y para el análisis y cuantificación de la VM, así como determinar los mecanismos de insuficiencia y la geometría del orificio regurgitante. Es de especial apoyo en la valoración intraoperatoria con la finalidad de corroborar la lesión anatómica y definir la severidad de las lesiones residuales.

\section{Doble salida de ventrículo derecho}

La doble salida de ventrículo derecho incluye un espectro anatómico extremadamente variado. Se requiere un entendimiento detallado de las variantes anatómicas para un diagnóstico correcto y la decisión del tipo de corrección quirúrgica, entre ellas la localización, tamaño, relación y distancia de la comunicación interventricular con las valvas semilunares, morfología conal, relación espacial de las grandes arterias y lesiones asociadas: obstrucciones a las vías de salida, anomalías de las válvulas AV, hipoplasia ventricular o patrones 
coronarios anormales. La ecocardiografía desempeña un papel vital en el diagnóstico: la Eco2D se ha utilizado tradicionalmente para planificar intervención quirúrgica, utilizando múltiples vistas ecocardiográficas separadas, y la Eco3D tiene la ventaja de incorporar profundidad a las imágenes con lo cual las válvulas AV, la comunicación interventricular y las grandes arterias pueden visualizarse en una sola proyección. ${ }^{13}$

\section{Función ventricular 3D}

La valoración de la función ventricular en CC no solo depende de la función izquierda, en algunas cardiopatías nos enfrentamos a un ventrículo derecho sistémico, como en la transposición corregida de grandes arterias o con presión elevada por obstrucción pulmonar o sobrecarga volumétrica (como sucede en el paciente posoperado de tetralogía de Fallot con insuficiencia pulmonar residual) o incluso a fisiología de ventrículo único, por lo que la cuantificación volumétrica tridimensional para la valoración de la función es una alternativa para el estudio y seguimiento de estos pacientes. ${ }^{14}$

El visualización del ventrículo izquierdo mediante Eco3D en pacientes con CC permite analizar volúmenes, función y análisis segmentario, así como el estudio de disincronía, sin embargo, aun cuando se ha demostrado la comparabilidad del Eco3D con la resonancia magnética, la medicina nuclear y la ventriculografía, no existen investigaciones suficientemente extensas que permitan establecer rangos de valores normales de estas estructuras en pacientes pediátricos, además de variaciones en el cálculo de volúmenes y función ventriculares debido a los cambios morfológicos de las cámaras inherentes a las CC. A pesar de esto, ha demostrado ser útil principalmente en las cardiopatías en las que, por la propia fisiopatología, el ventrículo izquierdo modifica su anatomía.

Hasta hace poco, la utilidad del Eco3D en la medición de volumen del ventrículo derecho (VD) y la fracción de eyección no había sido analizada debido a la complejidad de la morfología de este, así como a la dificultad técnica para la obtención de imágenes adecuadas. Recientemente se ha demostrado la utilidad de las mediciones volumétricas del VD en comparación con la imagen por resonancia magnética, mediante la técnica de adición de discos, usando un protocolo de reconstrucción multiplanar y detección automática de bordes, con la cual se ha identificado una excelente correlación entre las medidas hechas por Eco3D y aquellas por resonancia magnética. En pacientes con corazones univentriculares, el Eco3D ha demostrado obtener resultados comparables con la resonancia magnética en la obtención de imágenes, así como valores menores a los obtenidos por esta última en el cálculo de volúmenes y fracción de eyección. ${ }^{15}$ 


\title{
Evolución y resultados en el tratamiento de las cardiopatías congénitas a nivel mundial
}

\author{
Carlos Alva-Espinosa
}

El concepto de evolución proviene del término latino evolutio y hace referencia al verbo evolucionar y a sus efectos. En su acepción más amplia, la evolución es un proceso universal que consiste en el cambio. Nuestro planeta surgió y ha evolucionado a partir de la evolución del propio universo, lo primero hace 4600 millones de años, lo segundo tiene al menos 13700 millones de años. La vida en la Tierra surgió hace más de 3 mil millones de años y se ha desplegado en formas cada vez más complejas y diversas, sin embargo, este desarrollo evolutivo no había tenido dirección, propósito o sentido. Los cambios son producto de contingencias al azar ante la inmensidad del tiempo donde la selección natural ha sido ciega. Muy recientemente, a partir de la aparición del hombre, con su conciencia capaz de reflexionar sobre el pasado y proyectarlo al futuro, la evolución por primera vez tiene dirección. La humanidad apunta hacia una transformación de su entorno y de su propia vida para establecer el progreso humano, no sin retrocesos, pero paulatina y progresivamente modificando la historia natural. La medicina es un buen ejemplo de este proceso. Las intervenciones médicas se miden contra la evolución natural de la enfermedad, señaladamente la sobrevida.

La evolución en el conocimiento y tratamiento de las CC ha sido uno de los logros más espectaculares de la medicina. A mediados del siglo pasado, los niños con tetralogía de Fallot difícilmente alcanzaban la vida adulta. A partir de 1938, cuando el doctor Gross ${ }^{16}$ realizó la primera ligadura de un conducto arterioso con éxito, se inició un cambio en el pronóstico de los enfermos con CC. Desde entonces y a menos de 80 años de distancia, la posibilidad de que un niño con $\mathrm{CC}$ que recibe tratamiento llegue a la vida adulta es de $89 \% .{ }^{17}$ Un reflejo fiel del progreso en el tratamiento de estos enfermos es el hecho de que desde 2010 existen más adultos con CC que niños con esta anomalía. Del total de estos enfermos, $66 \%$ es adulto. ${ }^{18}$ A continuación se presentan algunos de los cambios más significativos en los resultados de los tratamientos de las CC, con el propósito fundamental de actualizar la conceptualización diagnóstica, terapéutica y pronóstica de este grupo de enfermos.

\section{Transposición de los grandes vasos (TGV)}

Es en el tratamiento de los recién nacidos con transposición de los grandes vasos (TGV) donde se demuestra con claridad el progreso en el tratamiento de estas lesiones. El ecocardiograma bidimensional es suficiente para hacer el diagnóstico (Figura 4). La TGV es una cardiopatía compleja, los niños presentan cianosis con manifestaciones de insuficiencia cardiaca en los primeros días de vida y sin tratamiento tienen muy alta mortalidad: $90 \%$ de los niños nacidos con TGV fallece al año de vida. ${ }^{19}$ En la actualidad, la operación de Jatene o switch arterial (la operación que corrige la TGV) tiene una mortalidad operatoria de 0 a $2.2 \%,,^{20}$ en tanto que la supervivencia a 15 años es de $97.6 \%$, con una calidad de vida prácticamente normal. ${ }^{21}$

\section{Comunicación interauricular}

La comunicación interauricular es una de las CC más frecuentes, consiste en el paso de sangre de la aurícula izquierda a la derecha a través del defecto; afecta principalmente a niñas, quienes cursan asintomáticas o con disnea ligera y palpitaciones. En la década de 1970, el doctor Campbell estimó una mortalidad de $50 \%$ en su historia natural. ${ }^{22}$ En 1955, cuando se iniciaba la cirugía a corazón abierto, Lillehei obtenía una mortalidad de $33 \%$ mediante la circulación cruzada padre/hija. ${ }^{23}$ Con el cierre quirúrgico actual, la supervivencia a largo plazo del cierre de la comunicación interauricular es de $99.6 \%{ }^{24}$ y no solo los resultados quirúrgicos son excelentes, con el advenimiento del intervencionismo percutáneo, la comunicación interauricular puede cerrarse sin circulación extracorpórea mediante un dispositivo colocado en el defecto por el cardiólogo intervencionista, con un éxito de $95.7 \%$, lo cual evita la bomba de circulación extracorpórea, el dolor y la cicatrización de la herida quirúrgica; por estas ventajas, el cierre por cateterismo es ahora el tratamiento de elección para la comunicación interauricular. ${ }^{25}$ 


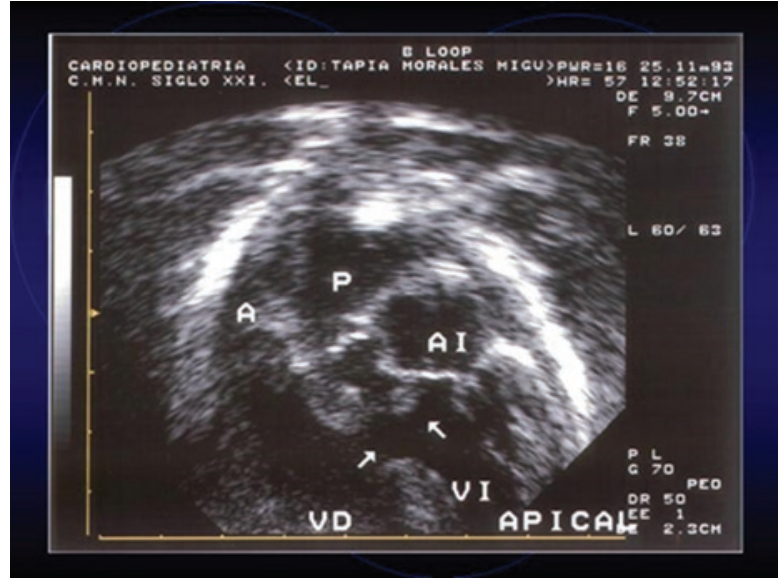

Figura 4. Ecocardiograma, abordaje apical. Transposición completa de las grandes arterias. Se observan las discordancias ventrículo-arteriales: del ventrículo derecho VD nace la aorta $(A)$ y del ventrículo izquierdo nace la pulmonar $(P)$. Existe comunicación interventricular subpulmonar (flecha horizontal) y estenosis subpulmonar (flecha vertical). $A I=$ aurícula izquierda, $V D=$ ventrículo derecho, $V I=$ ventrículo izquierdo.

\section{Tetralogía de Fallot}

La tetralogía de Fallot es la CC con cianosis más frecuente y es una de las más investigadas. Su combinación de lesiones es bien conocida: estenosis pulmonar, comunicación interventricular, cabalgamiento de la aorta (sobre el septum interventricular) e hipertrofia ventricular derecha. En ocasiones es necesario el cateterismo para hacer el diagnóstico (Figura 5). La cianosis es el dato cardinal en el diagnóstico, la cual se incrementa con el tiempo. En 1978, Kirklin et al. estimaron que $50 \%$ de los enfermos que nace con tetralogía de Fallot no operados muere en los primeros años de vida y difícilmente alguno sobrevive más allá de los 30 años. ${ }^{26} \mathrm{El}$ tratamiento es quirúrgico. Una mejor comprensión de la enfermedad, el refinamiento en el proceso diagnóstico y el progreso en la tecnología y técnicas quirúrgicas permitió operar a estos niños a edades cada vez más tempranas: la edad recomendada para la corrección es a los seis meses de vida. La mortalidad operatoria actual es de $2 \% 0$ menor $^{27,28}$ y el seguimiento a largo plazo, a 40 años, ha demostrado una supervivencia de $77.5 \%$, con una calidad de vida prácticamente normal en la mayoría de los casos. ${ }^{29}$

\section{Mortalidad operatoria global}

Existe una reciente y excelente publicación sobre los resultados quirúrgicos de todas las $\mathrm{CC}$ operadas de 1997 a 2015 en Inglaterra, con un poco más de

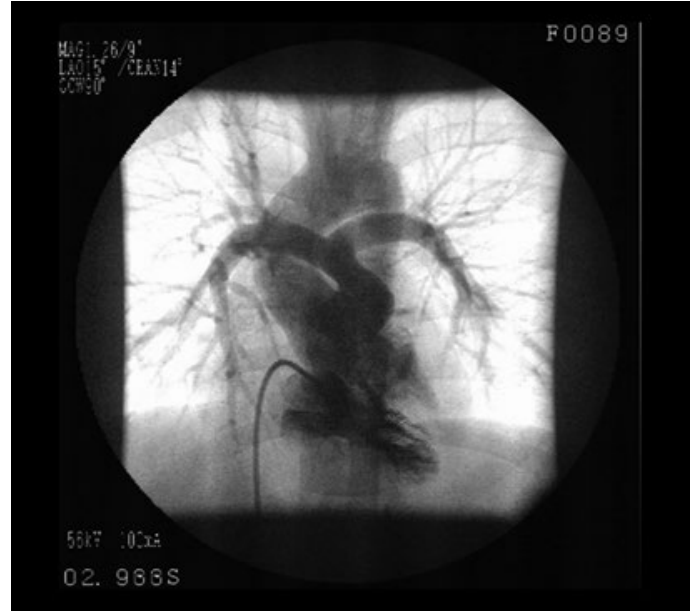

Figura 5. Angiocardiograma selectivo en el ventrículo derecho en proyección posteroanterior con angulación craneal de $30^{\circ}$ de un niño de seis años con tetralogía de Fallot. Se observa el ventrículo derecho hipertrófico con estenosis infundibular y valvular. El anillo valvular, el tronco y las ramas de la arteria pulmonar son de buen calibre. Existe opacificación simultánea de la aorta, moderadamente dilatada que describe arco aórtico derecho. Es un enfermo con tetralogía de Fallot con anatomía favorable.

57 mil enfermos operados. La mortalidad operatoria en su conjunto fue de $2.93 \%$. Cuando los resultados se separan en cirugía urgente, la mortalidad es de $6.5 \%$; cuando es electiva, la mortalidad es de $1.65 \%{ }^{30}$

\section{Papel de la cardiología intervencionista en el tratamiento de las cardiopatías congénitas}

Desde el inicio de la cardiología intervencionista en 1953 por Rubio Álvarez et al. para tratar un caso de estenosis pulmonar con cateterismo en México ${ }^{31}$ a la fecha del presente artículo, la evolución y aplicación de esta técnica en las $\mathrm{CC}$ ha sido exponencial. A partir de una experiencia de 39 años, un destacado artículo reciente resume este progreso: el cateterismo intervencionista como tratamiento inicial pasó de 5.8 a $25.9 \%$ y de ser el único tratamiento pasó de 4.8 a $24 \%$; adicionalmente, la edad promedio de 3.4 años bajó a 0.6 años. Sin embargo, esto no ha significado una disminución de las intervenciones quirúrgicas, que aumentaron de 1717 en 1997 a 5299 en un trabajo inglés. ${ }^{30}$ La explicación del crecimiento, tanto de la cardiología intervencionista como de la cirugía, es consistente con el aumento del número de enfermos y la mayor disposición de opciones terapéuticas que hace algunas décadas no estaban disponibles. 


\section{Prevalencia de las cardiopatías congénitas en los adultos}

Como consecuencia de la disminución en la mortalidad de los enfermos con CC, en Canadá la prevalencia en los niños se ha incrementado $22 \%$, en tanto que en los adultos este efecto ha sido más pronunciado: la prevalencia ascendió $85 \%$ entre 1985 y 2000. ${ }^{32}$

En 2016, Gilboa et al. publicaron el número total de enfermos vivos estimados con CC en Estados Unidos: 2.4 millones, de los cuales 1.4 millones son adultos. ${ }^{33}$

En México, en un cálculo conservador realizado en 2006 estimamos 300 mil adultos vivos con CC, con un incremento anual de 15 mil casos, ${ }^{34}$ de modo que para 2018 podría haber cerca de medio millón de adultos con CC en México.

Ante el incremento significativo de enfermos con CC, los países desarrollados se han organizado para enfrentar el reto de atender la compleja transición de los adolescentes con CC y su ingreso a la vida adulta. Esto requiere un equipo multidisciplinario. Con este desafío en mente, en 1991 se fundó la Canadian Adult Congenital Heart Net Work, con el propósito explícito de atender a estos enfermos, mientras que en 1993, en Europa se fundó The Jane Somerville Fundation, con el mismo objetivo, la cual evolucionó a la Grown Up Congenital Heart Disease de la European Society of Cardiology. En 1994 se crearon la Adult Congenital Heart Association y la International Society for Adult Congenital Heart Disease. En esos países también se creó la subespecialidad en cardiopatías congénitas en el adulto. En México, si bien en el Hospital de Cardiología del Centro Médico Nacional Siglo XXI, en el Instituto Nacional de Cardiología y en otros centros existen cardiólogos dedicados a la atención de estos enfermos, no se cuenta con la subespecialidad.

El creciente número de enfermos con $\mathrm{CC}$, que paradójicamente refleja el éxito terapéutico en las últimas décadas, implica un reto a la salud pública. Será necesario preparar en el país más especialistas en CC en niños y adultos para hacer frente con calidad y eficiencia a este creciente grupo de enfermos. 


\title{
La mujer embarazada con cardiopatía congénita
}

\author{
Lucelli Yáñez-Gutiérrez
}

El avance en el tratamiento de las $\mathrm{CC}$ ha permitido que los pacientes tengan una supervivencia mayor $y$ nueve de cada 10 lleguen a la edad adulta; $50 \%$ aproximadamente es del sexo femenino y en algún momento se planteará la posibilidad de la maternidad y deseará llevar a término un embarazo, que se considerará de alto riesgo.

La hipertensión arterial sistémica es la complicación cardiaca más frecuente en el embarazo y la CC es el trastorno cardiovascular más frecuente. Se estima que pueden ser afectadas una de cada 156 mujeres embarazada, grupo en el cual se ha registrado una mortalidad de dos por cada 100000 embarazadas y una mortalidad global aproximada de $5 \%$. Por lo anterior, las enfermedades cardiacas son la principal causa de mortalidad materna durante el embarazo. ${ }^{35} \mathrm{El}$ embarazo per se es un estado que provoca cambios en el sistema cardiovascular para cubrir las demandas de la madre y el feto. La debida atención materno-fetal es un reto que hace imprescindible la integración de equipos multidisciplinarios capacitados para la atención de estas pacientes.

En México se estima que el inicio de la vida sexual ocurre aproximadamente alrededor de los 12 años, por lo tanto es importante iniciar en forma temprana con la sensibilización de los padres y las niñas y adolescentes cardiópatas para conocer su enfermedad y orientar sobre la morbimortalidad de las intervenciones a las que se someterán en el futuro, el riesgo de complicaciones al llegar a la edad adulta, la esperanza de vida según el tipo de cardiopatía y la probable necesidad de nuevas cirugías en la edad adulta, el riesgo de herencia, así como el uso de fármacos cardiovasculares que pueden ocasionar malformaciones fetales, haciendo énfasis en el uso de anticoagulación oral y el riesgo de fetopatía inducida por este grupo de fármacos.

Se deben proponer métodos de planificación familiar en forma temprana para promover una vida sexual responsable y sin riesgo de infecciones de transmisión sexual o embarazos no deseados, y en forma conjunta con el servicio de ginecología se debe elegir el método que no implique mayor riesgo. Es importante dar a conocer cuáles podrían ser los métodos de planificación que representan las mejores alternativas. Por ejemplo, los hormonales incrementan el riesgo de
Tabla 1. Cambios hemodinámicos durante el embarazo

Aumento del gasto cardiaco de 30 a $50 \%$

Aumento del volumen sanguíneo 18 a $25 \%$

Disminución de la diferencia auriculoventricular de oxígeno

Aumento de la contractilidad miocárdica

Aumento de la frecuencia cardiaca de $20 \%$

(10 a 20 latidos por minuto)

Aumento del volumen plasmático de 40 a $50 \%$

Disminución de la presión arterial sistémica

Aumento de la presión de pulso

Disminución de la diastólica > disminución de la sistólica

Aumento del flujo regional

(útero, glándula mamaria, riñón, piel)

Disminución de las resistencias vasculares

(sistémicas y pulmonares)

$\leftrightarrow$ Presión venosa central

$\leftrightarrow$ Cuña

Hipercoagulabilidad

eventos tromboembólicos, alteran el metabolismo de los anticoagulantes orales, fallan cuando se tienen que utilizar antibióticos y pierden su efecto al utilizar bosentán. El dispositivo intrauterino representa un riesgo bajo de bacteremia o de endocarditis infecciosa y al momento de su colocación $5 \%$ de las pacientes puede presentar un fenómeno vasovagal derivado de la manipulación del cérvix. Los menos recomendados son los métodos de barrera, por la alta probabilidad de falla. En cuanto a los métodos definitivos se podrán utilizar ciertos implantes intratubarios que pueden ser removidos cuando se planee un embarazo. ${ }^{36}$

La atención de estas pacientes se debe hacer en forma integral, con equipos conformados por cardiólogos pediatras, cardiólogos especializados en la atención de adultos con CC, ginecoobstetras perinatólogos, así como otros especialistas entrenados en complicaciones.

Un aspecto importante por considerar son los cambios que modifican el estado cardiovascular en forma fisiológica durante un embarazo: se produce un incremento hasta de $50 \%$ del gasto cardiaco y del volumen 
Tabla 2. Mortalidad materna según la clase funcional

\begin{tabular}{lllr}
\hline Clase & & Manifestaciones & Mortalidad (\%) \\
\hline I & Asintomática & Sin limitación de actividad física & 2.5 \\
II & Disnea grandes esfuerzos & Actividad ordinaria produce palpitaciones, disnea o angina & $5.7-10.5$ \\
II-III & Disnea medianos esfuerzos & Pocas actividades físicas & $10-19$ \\
III & Disnea de mínimos esfuerzo & Normalidad tan solo en reposo, imposible actividades físicas menores & $19-27$ \\
IV & Disnea de reposo & Insuficiencia cardiaca o angina de reposo & $40-50$ \\
\hline
\end{tabular}

Tabla 3. Manejo de la paciente según el riesgo

\begin{tabular}{|c|c|c|c|}
\hline No incrementa & Incremento ligero & Incremento moderado & Alto riesgo \\
\hline 1 o 2 visitas al cardiólogo & Visita cada 3 meses & Revisión cada 1 a 2 meses & Seguimiento continuo \\
\hline $\begin{array}{l}\text { No reparadas } \\
\text {-Estenosis pulmonar } \\
\text {-Persistencia del conducto arterioso } \\
\text {-Prolapso de la válvula mitral }\end{array}$ & $\begin{array}{l}\text { No reparadas } \\
\text {-Comunicación interatrial } \\
\text {-Comunicación } \\
\text { interventricular }\end{array}$ & $\begin{array}{l}\text { No reparadas } \\
\text {-Tetralogía de Fallot } \\
\text {-Ventrículo derecho sistémico }\end{array}$ & \multirow{2}{*}{$\begin{array}{l}\text {-Interrupción del embarazo } \\
\text {-Hipertensión arterial pulmonar severa } \\
\text {-Fracción de expulsión del ventrículo } \\
\text { izquierdo }<30 \% \\
\text {-Clase funcional III-IV } \\
\text {-Cardiomiopatía periparto } \\
\text {-Coartación severa } \\
\text {-Síndrome de Marfán con dilatación } \\
\text { aórtica > } 45 \text { mm } \\
\text {-Aorta bivalva con dilatación > } 50 \text { mm }\end{array}$} \\
\hline $\begin{array}{l}\text { Reparadas } \\
\text {-Comunicación interatrial } \\
\text {-Comunicación interventricular } \\
\text {-Persistencia del conducto arterioso } \\
\text {-Conexión venosa pulmonar anómala }\end{array}$ & $\begin{array}{l}\text { Reparadas } \\
\text {-Tetralogía de Fallot }\end{array}$ & $\begin{array}{l}\text { Reparadas } \\
\text {-Cirugía de Fontan }\end{array}$ & \\
\hline
\end{tabular}

sanguíneo total, se incrementa la frecuencia cardiaca y la presión arterial sistémica tiende a disminuir en los dos primeros trimestres y también ocurren cambios respiratorios, como el incremento de $50 \%$ de la ventilación por minuto y aproximadamente un incremento de $20 \%$ en el consumo de oxígeno, con disminución de $40 \%$ de la capacidad residual funcional (Tabla 1). ${ }^{37}$ Asimismo, ocurren cambios estructurales reversibles que pueden ser mal tolerados en las mujeres con cardiopatía, como hipertrofia miocárdica, dilatación de cavidades cardiacas, insuficiencia valvular, depresión de la contractilidad y discreta dilatación de la raíz aórtica. No debe olvidarse que durante el embarazo se incrementan los fenómenos proarrítmicos y protrombóticos.

El reto es doble, se debe considerar el riesgo materno y el riesgo fetal. Por lo anterior, diversos estudios multicéntricos han establecido los factores de riesgo para morbimortalidad materna y fetal:

- Materna: historia de arritmias, uso de más de tres fármacos cardiovasculares, clase funcional antes del embarazo, cardiopatía con obstrucción izquierda, insuficiencia valvular severa, prótesis mecánica (anticoagulación) y cianosis.

- Fetal: gestación múltiple, historia de tabaquismo materno, cardiopatía cianógena, presencia de prótesis mecánica y uso de más de tres fármacos cardiovasculares. ${ }^{38}$
La Organización Mundial de la Salud (OMS) estratifica los riesgos en el embarazo de la siguiente forma:

- OMS I (riesgo materno de eventos cardiacos de 2.5 a $5 \%$ ): cardiopatías leves (por ejemplo, estenosis pulmonar, conducto arterioso, prolapso del VM), defectos cardiacos reparados exitosamente (cierre de comunicación interatrial, interventricular o conducto arterioso, conexión venosa pulmonar anómala) y extrasístoles auriculares o ventriculares aisladas

- OMS II (riesgo materno de eventos cardiacos de 5.7 a $10.5 \%$ ): comunicación interatrial o interventricular no operada, tetralogía de Fallot operada y la mayoría de las arritmias.

- OMS II-III (riesgo materno de eventos cardiacos de 10 a $19 \%$ ): disfunción ventricular izquierda ligera, miocardiopatía hipertrófica, otras valvulopatías, síndrome de Marfán sin dilatación aórtica, coartación aórtica reparada

- OMS III (riesgo materno de eventos cardiacos de 19 a $27 \%$ ): prótesis mecánica, ventrículo derecho sistémico y fisiología de Fontan.

- OMS IV (riesgo materno de eventos cardiacos de 40 a $100 \%$ ): hipertensión arterial pulmonar por cualquier causa, disfunción ventricular sistémica grave (FEVI < $30 \%$ ), miocardiopatía periparto previa, obstrucción izquierda grave (mitral o aórtica), síndrome de Marfán con aorta dilatada 
(> $45 \mathrm{~mm}$ ) o dilatación mayor de $50 \mathrm{~mm}$ en aorta bivalva, coartación aórtica grave ${ }^{5}$ (Tabla 2).

Cuando se considere someter a la mujer embarazada a trabajo de parto, no deben olvidarse los cambios hemodinámicos que ocurren por el dolor y la ansiedad y que con cada contracción uterina se incrementa $50 \%$ la frecuencia cardiaca y el gasto cardiaco, es decir, 300 a $400 \mathrm{~mL}$ más, y que el gasto cardiaco aumenta al momento de descomprimir la vena cava inferior, por transferencia de sangre desde el útero contraído, por lo tanto no se podrá considerar como opción en las pacientes con cortocircuitos no resueltos o residuales (por ejemplo, comunicación interatrial amplia o conducto arterioso) y en algunos casos se deberá proponer cesárea con profilaxis para endocarditis y manejo adecuado de la volemia y ventilación. Con base en estos estudios se debe definir el seguimiento de estas pacientes y cómo se dará término al embarazo (Tabla 3).

Algunos procedimientos terapéuticos podrán llevarse a cabo con relativa seguridad, como estudios de imagen (radiografías, tomografías o angiografías) 0 intervencionistas (angioplastias y valvuloplastias), no así la cirugía cardiaca, ya que la derivación cardiopulmonar compromete la viabilidad fetal y el riesgo de pérdida fetal es de $30 \% .^{39}$

Los aspectos más relevantes en relación con la mujer embarazada con CC pueden resumirse de la siguiente forma:

- Conocer los cambios hemodinámicos fisiológicos ocurridos durante el embarazo facilita la detección de complicaciones en el contexto de mujeres que tienen $\mathrm{CC}$.

- El riesgo materno o fetal está relacionado directamente con el tipo de cardiopatía, el tipo de reparación quirúrgica o las complicaciones tardías inherentes a las cardiopatías.

- La atención de mujeres embarazadas que tienen CC debe proporcionarse por un equipo multidisciplinario.

- Es obligación del cardiólogo pediatra y del cardiólogo experto en CC orientar a la mujer con cardiopatía que se enfrenta a la decisión de un embarazo, el cual con un buen manejo podrá llevar a buen término. 


\title{
La realidad de las cardiopatías congénitas en México
}

\author{
Horacio Márquez-González
}

No hay mayor incertidumbre que el desconocimiento del problema, lo que puede aplicarse al tema de las CC en México. Anualmente esperamos que 15000 a 18000 neonatos tengan una malformación cardiaca, sin embargo, es una estimación de la incidencia de otros países extrapolada al número de nacimientos anuales en México. Realmente nuestro sistema de información no puede precisar las unidades epidemiológicas básicas como frecuencia, incidencia y prevalencia de las CC en niños y adultos a nivel nacional ni por entidad federativa.

La centralización de los hospitales retarda y limita el acceso a los recursos. Hasta 2009 existían en nuestro país nueve centros certificados en la atención de las CC (siete en la Ciudad de México), con la característica adicional de que sus tareas eran exclusivamente especializadas en las áreas pediátricas o cardiológicas, por lo que obligadamente al cumplir la mayoría de edad era necesario el envío a otros hospitales. ${ }^{40}$ En este intervalo, factores como el tipo de afiliación a un área determinada del sector salud, el tiempo transcurrido para la primera consulta al nuevo hospital y la disponibilidad del recurso económico fueron responsables de que el proceso de atención se viera interrumpido. Sin embargo, actualmente se encuentran operando nuevos hospitales que se espera alcancen la curva de especialización en la siguiente década.

Otra realidad es que existe un retraso en la detección temprana. Aunque el diagnóstico fetal de las CC actualmente puede realizarse por ginecólogos que tienen formación de posgrado en salud materno-fetal, aún no hay una adecuada distribución de estos profesionales en los sitios donde debería realizarse la labor de tamizaje, además, el mal uso del ultrasonido en manos de profesionales no preparados condiciona que la dirección y la oportunidad de diagnóstico se vea mermada.

También es real que el pediatra clínico no tiene total destreza y necesita la interacción con el cardiólogo pediatra, quien habitualmente no desempeña sus labores en el segundo nivel de atención, por lo que el proceso de referencia puede representar una verdadera dificultad para la atención en algunas entidades federativas.

\section{Clínicas de enlace}

Las clínicas de transición o de enlace son una realidad en algunos países donde está establecido el mínimo de competencias (aseguradas por cursos con aval universitario no menores a un año) que deben adquirir el cardiólogo clínico y el cardiólogo pediatra para asegurar que el enlace se complete adecuadamente y se permita un seguimiento por un profesional médico que orqueste la cobertura de las necesidades en las futuras etapas de la vida.

\section{El adulto con CC en México}

En México son pocos los hospitales con una clínica de enlace en CC que puedan aportar información sobre el estado actual del paciente cardiópata en la etapa adulta. En el Hospital de Cardiología del Centro Médico Nacional Siglo XXI, Instituto Mexicano del Seguro Social, se cuenta con una de ellas. En 2017 publicó una casuística de cinco años. ${ }^{41}$ En relación con la población adulta, de 3483 enfermos confirmados con CC, $25.6 \%$ fue diagnosticado en la etapa adulta y las tres principales alteraciones fueron la comunicación interauricular, la interventricular y la persistencia del conducto arterioso. Este centro hospitalario tiene la particularidad de ser de tercer nivel y cubrir la zona centro y suroeste de la población derechohabiente del Instituto Mexicano del Seguro Social en el país. El análisis por entidades federativas demostró que en Chiapas el diagnóstico de CC se realizó durante la adultez en $33 \%$ de los pacientes, en Oaxaca en $19 \%$ y en Guerrero en $17 \%$. En cuanto a la mujer adulta con CC, $18 \%$ fue diagnosticada en su primer embarazo y $17 \%$ ya sabía que era portadora de esta malformación, a pesar de lo cual se embarazó sin ninguna estrategia para la planificación familiar ni para la resolución del embarazo. Estos datos deben ser considerados como alarma, sobre todo en lo que concierne a la mortalidad materna y en la primera infancia, por la ya conocida asociación entre la madre con $\mathrm{CC}$ y la prematurez 0 asfixia del producto en $50 \%$ de los casos.

También se publicaron resultados del estado de Chiapas con base en los datos reportados en la Encuesta Intercensal de 2015. ${ }^{42}$ La población total del estado fue de 5217908 habitantes (2 681187 mujeres), de los cuales 626148 derechohabientes (12.4\%) se encontraron afiliados al régimen ordinario del Instituto Mexicano del Seguro Social. Se encontró que uno de cada 2000 adultos tenía una CC no diagnosticada, 1.8 de cada 2000 mujeres afiliadas en etapa reproductiva tenía una CC y la desconocía, lo que significa que uno de cada 10 embarazos complicados pueden estar asociados con esta causa. 


\section{Conclusiones}

1. La ecocardiografía es la herramienta diagnóstica más útil y práctica en CC. Los avances con la modalidad tridimensional, transtorácica y transe sofágica aportan beneficios adicionales en el diagnóstico de las CC, tanto en su estructura y función, como en la planeación del tratamiento y evaluación transoperatoria.

2. En el tratamiento de las CC, la mortalidad operatoria y a largo plazo han disminuido significativamente. Actualmente, $89 \%$ de los enfermos llega a la vida adulta. Del total de los enfermos, dos tercios son adultos.

3. El papel de la cardiología intervencionista es cada vez más importante, sin embargo, también el número de enfermos que requieren cirugía va en aumento.

4. La mujer con CC en edad reproductiva debe contar con un plan de control natal y una evaluación de riesgo para cuando decida embarazarse.

5. Durante el embarazo se producen cambios estructurales y funcionales que implican un reto adicional, tanto para la mujer con CC como para el feto.

6. La atención adecuada de la mujer embarazada con CC requiere el trabajo de un equipo multidisciplinario.

7. En México es importante conocer la incidencia, prevalencia y frecuencia real de las CC tanto en niños como en adultos, a través de un sistema de registro nacional que permita identificar de forma objetiva el problema.

8. Es necesaria la formación de especialistas clínicos, quirúrgicos e intervencionistas en $\mathrm{CC}$ en niños y adultos, para responder a la creciente demanda de atención de estos enfermos.

\section{Bibliografía}

1. Del Pasqua A, Sanders SP, De Zorzi A, Toscano A, Lacobelli R, Pierli C, et al. Impact of three-dimensional echocardiography in complex congenital heart defect cases: the surgical view. Pediatr Cardiol. 2009; 30:293-300.

2. Jenkins $C$, Monaghan M, Shirali G, Guraraja R, Marwick TH. An intensive interactive course for 3D echocardiography: is "crop till you drop" an effective learning strategy? Eur J Echocardiogr. 2008;9:373-380.

3. Srivastava S, Printz BF, Geva T, Shirali GS, Weinberg PM, Wong PC, et al. Task force 2: pediatric cardiology fellowship training in noninvasive cardiac imaging: endorsed by the American Society of Echocardiography and the Society of Pediatric Echocardiography. J Am Soc Echocardiogr. 2015;66:687-698.

4. Von Ramm OT, Smith SW. Real time volumetric ultrasound imaging system. J Digit Imaging. 1990;3:261-266.

5. Vargas-Barrón J, Romero-Cárdenas A, Espínola N, Roldán Gómez FJ, Vázquez-Antona CA, Attie F, et al. Three-dimensional echocardiography in adult congenital heart disease. En: Nanda NC, Sorrell VL, editors. Atlas of three-dimensional echocardiography. EE. UU.: Futura Publishing; 2002.
6. Vázquez-Antona CA, Muñoz L, Erdmenger J, Roldán J, Vargas J. Ecocardiografía tridimensional en la evaluación de los defectos cardiacos congénitos. Portuguese J Card. 2004;23:60.

7. Vázquez-Antona, CA. Ecocardiografía tridimensional en cardiopatías congénitas. En: Martínez-Ríos MA, Damas De Los Santos F, Lorenzo-Negrete JA, editores. Contribuciones del Instituto Nacional de Cardiología Ignacio Chávez. México: Permanyer; 2014.

8. De Castro S, Caselli S, Papetti F, Ventriglia F, Giardina A, Cavaretta E, et al: Feasibility and clinical impact of live three-dimensional echocardiography in the management of congenital heart disease. Echocardiography. 2006;23:553-556

9. Simpson J, Lopez L, Acar P, Friedberg M, Khoo N, Ko H, et al. Three-dimensional echocardiography in congenital heart disease: an expert consensus document from the European Association of Cardiovascular Imaging and the American Society of Echocardiography. Eur Heart $J$ Cardiovasc Imaging. 2016;17:1071-1097.

10. Roldán FJ, Vargas-Barrón J, Vázquez-Antona CA, Castellanos LM, Erdmenger-Orellana J, Romero-Cárdenas A, et al. Three dimensional transesophageal echocardiography of atrial septal defects. Cardiovasc Ultrasound. 2008;6:38.

11. Balzer J, Van-Hall S, Böring YC, Kelm M. New role of echocardiography in the Cath Lab: novel approaches of peri-interventional 3D echocardiography. Curr Cardiovasc Imaging Rep. 2013;6:445-453.

12. Kutty S, Colen TM, Smallhorn JF. Three-dimensional echocardiography in the assessment of congenital mitral valve disease. J Am Soc Echocardiogr. 2014;27:142-154.

13. Pushparajah K, Barlow A, Tran VH, Miller OI, Zidere V, Vaidyanathan B, et al. A systematic three-dimensional echocardiographic approach to assist surgical planning in double outlet right ventricle. Echocardiography. 2013;30:234-238.

14. Renella P, Marx GR, Zhou J, Gauvreau K, Geva T. Feasibility and reproducibility of three-dimensional echocardiographic assessment of right ventricular size and function in pediatric patients. J Am Soc Echocardiogr. 2014;27:903-910

15. Jurcut R, Giusca S, La-Gerche A, Vasile S, Ginhina C, Voigt JU. The echocardiographic assessment of the right ventricle: what to do in 2010 ? Eur J Echocardiogr. 2010;11:81-96.

16. Gross RE, Hubbard JP. Surgical ligation of patent ductus arteriosus. Report of first successful case. JAMA. 1939;112:129.

17. Moons P, Bovijn L, Budts W, Belmans A, Gewillig M. Temporal trends in survival to adulthood among patients born with congenital heart disease from 1970 to 1992 in Belgium. Circulation. 2010;122:2264-2272.

18. Marelli AJ, Ionescu-Ittu R, Mackie AS, Guo L, Dendukuri N, Kaouache M. Lifetime prevalence of congenital heart disease in the general population from 2000 to 2010. Circulation. 2014;130:749-756.

19. Liebman J, Cullum L, Belloc NB. Natural history of transposition of the great arteries. Circulation. 1969;40:237-262.

20. Frazer C. Resultados de switch para TGV. Semin Thorac Cardiovasc Surg Pediatr Card Surg Annu. 2017;20:38-42.

21. Shim MS, Jun TG, Yang JH, Park PW, Kang IS, Huh J, et al. Current expectations of the arterial switch operation in a small volume center: a 20-year, single-center experience. Cardiothorac Surg. 2016;11:34.

22. Campbell M. Natural history of atrial septal defect. $\mathrm{Br}$ Heart J. 1970;32:820-835

23. Lillehei CW, Cohen M, Warden HE, Varco RL. The direct vision intracardiac correction of congenital anomalies by controlled cross circulation; results in thirty-two patients with ventricular septal defects, tetralogy of Fallot, and atrioventricularis communis defects. Surgery. 1955;38:11-29.

24. Téllez-De Peralta G. Historia de la cirugía cardiovascular. En: Tratado de cirugía cardiovascular. España: Sociedad Española de Cardiología; 1999.

25. Moore JW, Vincent RN, Beekman RH, Benson L, Bergersen L, Holzer R et al. Procedural results and safety of common interventional procedures in congenital heart disease: initial report from the National Cardiovascular Data Registry. J Am Coll Cardiol. 2014;64:2439-2451.

26. Bertranou EG, Blackstone EH, Hazelrig JB, Turner ME, Kirklin JW. Life expectancy without surgery in tetralogy of Fallot. Am J Cardiol. 1978; 42:458-466.

27. Caruana M, Grech V. A first population-based long-term outcome study in adults with repaired tetralogy of Fallot in Malta. Congenit Heart Dis. 2017;12:301-308.

28. Starr JP. Tetralogy of Fallot: yesterday and today. World J Surg. 2010;34:658-668.

29. Cuypers JA, Menting ME, Konings EE, Opić P, Utens EM, Helbing WA, et al. Unnatural history of tetralogy of Fallot: prospective follow-up of 40 years after surgical correction. Circulation. 2014;130:1944-1953. 
30. Kempny A, Dimopoulos K, Uebing A, Diller GP, Rosendahl U, Belitsis $G$, et al. Outcome of cardiac surgery in patients with congenital heart disease in England between 1997 and 2015. PLoS One. 2017; 12:e0178963.

31. Rubio-Álvarez V, Limón RL. Valvulotomía intracardiaca por medio de un catéter. Arch Inst Cardiol Mex. 1953;23:183-192.

32. Marelli AJ, Therrien J, Mackie AS, lonescu-Ittu R, Pilote L. Planning the specialized care of adult congenital heart disease patients: from numbers to guidelines; an epidemiologic approach. Am Heart J. 2009;157:1-8.

33. GilboaSM, DevineOJ, KucikJE, OsterME, Riehle-ColarussoT, NembhardWN, et al. Congenital heart defects in the United States: estimating the magnitude of the affected population in 2010. Circulation. 2016;134:101-109.

34. Alva-Espinosa C. Ante el adulto con cardiopatía congénita. Arch Cardiol Mex. 2006;76:57-61.

35. Knight M, Nair M, Tuffnell D. Saving lives, improving mothers' care. EE. UU.: University of Oxford; 2016.

36. Canobbio MM, Perloff J, Rapkin AJ. Gynecological health of females with congenial heart disease. Int J Cardiol. 2005;98:379-387.

37 Uebing A, Steer P, Yentis S, Gatzoulis M. Pregnancy and congenital heart disease. BMJ. 2006;332:401-406.
38. Van Hagen I, Boersma E, Johnson M. Global cardiac risk assessment in the registry of pregnancy and cardiac disease: Results of a registry from the European Society of Cardiology. Eur J Heart Fail. 2016;18:523- 533.

39. Regitz-Zagrosek V, Roos-Hesselink JW, Bauersachs J, Blomström-Lundqvist C, Cif́ková R. De Bonis M, et al. 2018 ESC Guidelines for the management of cardiovascular diseases during pregnancy. Eur Heart J. 2018;39(34): 3165-3241.

40. Calderón-Colmenero J, De La Llata M, Vizcaíno A, Ramírez-Marroquín S, Bolio-Cerdán A, Alva C, et al. Medical and surgical health care for congenital heart disease: a panoramic vision of the reality in Mexico. Inquiry 2009. Rev Invest Clin. 2011;63;344-352.

41. Márquez-González H, Yáñez-Gutiérrez L, Rivera-May JL, López-Gallegos D, Almeida-Gutiérrez E. Demographic analysis of a congenital heart disease clinic of the Mexican Institute of Social Security, with special interest in the adult. Arch Cardiol Mex. 2017;pii:S1405-9940.

42. Instituto Nacional de Estadística y Geografía. Principales resultados de la Encuesta Intercensal 2015. Estados Unidos Mexicanos. México: INEGI; 2015. Disponible en: http://internet.contenidos.inegi.org.mx/contenidos/productos/prod serv/contenidos/espanol/bvinegi/productos/nueva_estruc/702825078966.pdf 